\title{
The Relationship between Higher Left Ventricular Mass Index and Plasma Total Homocysteine Levels in patients with Type 2 Diabetes Mellitus
}

\author{
Harun Kundi ${ }^{1}$, $\underline{\text { Ihsan Ateș }}^{2}$, Nisbet Yılmaz ${ }^{2}$, Ender Örnek ${ }^{1}$ \\ ${ }^{1}$ Ankara Numune Education and Research Hospital, Department of Cardiology, Ankara, Turkey \\ ${ }^{2}$ Ankara Numune Education and Research Hospital, Department of Internal Meicine, Ankara, Turkey
}

\section{Objectives:}

\section{Methods:}

\section{Results:}

\section{References:}

The aim of the present study was left ventricular mass index (LVMI) in type 2 diabetes mellitus (T2DM) patients may be related increased circulating homocysteine (Hcy) levels.

The study group comprised 130 patients, and all patients were newly diagnosed with diabetes and were not taking any medication. Measures of left ventricular mass (LVM) were assessed using Devereux formula and left ventricular mass index (LVMI) was calculated as LVM divided by body surface area. Hcy levels were measured using high-performance liquid chromatography.

Forty five patients $(34.6 \%)$ of all patients with higher LVMI. In univariate logistic regression analyses age, creatinine and Hcy count all significantly associated with higher LVMI. When these 3 variables were included in a multivariate regression modeling Hcy levels were found to be significantly associat-ed with the higher LVMI

Hyperhomocysteinemia (Hhcy) can be a predictor of in T2DM patients. It can be easily used in this area, because it is easily available, inexpensive and practical.

1. Rydén, Lars, et al. "Guidelines on diabetes, pre-diabetes, and cardiovascular diseases: executive summary The Task Force on Diabetes and Cardiovascular Diseases of the European Society of Cardiology (ESC) and of the European Association for the Study of Diabetes (EASD)." European heart journal 28.1 (2007): 88-136.

2. Bagherzadeh, Ataollah, et al. "Association of cardiac autonomic neuropathy with arterial stiffness in type 2 diabetes mellitus patients." Journal of diabetes and metabolic disorders 12 (2013): 55.

3.Weiss, Norbert, et al. "Endothelial dysfunction and atherothrombosis in mild hyperhomocysteinemia." Vascular Medicine 7.3 (2002): 227-239. 